\title{
Prognostic Implication of Stress Induced Hyperglycemia in Non Diabetic Patients with Acute Coronary Syndrome
}

\author{
Sanjay Tukaram Thorat ${ }^{1}$, Radhika Ratanlal Bajaj², Vaibhav Vijay Rane ${ }^{3}$, \\ Avanti Jayant Damle ${ }^{4}$, Nikhil Dilip Patil ${ }^{5}$, Pankaj Nivrutti Pawar ${ }^{6}$ \\ 1,2, 3, 4, 5, 6 Department of Medicine, Krishna Institute of Medical Sciences Deemed to Be University, \\ Karad, Maharashtra, India.
}

\section{ABSTRACT}

\section{BACKGROUND}

Hyperglycemia is a common finding during the admission of non-diabetic patients with acute coronary syndrome. It is very essential to differentiate this stress hyperglycemia from the hyperglycemia in latent diabetics whose diabetes may have been unmasked by the stress of acute coronary syndrome (ACS). We conducted this study to evaluate stress hyperglycemia in non-diabetic patients with ACS.

\section{METHODS}

We conducted this cross sectional study in the Department of Medicine, KIMS Hospital, Karad, over a period of one year. Non-diabetic patients with HbA1c $<6.5$ admitted in our hospital with ACS were included in the study. We included a total of 100 patients in our study, their detailed history, clinical examination and lab evaluation was done. We divided the patients into two groups, Group A were normoglycaemic patients and Group B were acute phase hyperglycemic patients with blood sugars more than 160 . Correlation between the blood sugar levels and outcome of the patients was done.

\section{RESULTS}

Mean age was $65.23 \pm 21.05$ years. No significant difference in risk factor distribution in the two groups was seen. ( $p>0.05$ ) We had 47 cases of unstable angina (UA - 47\%) followed by 43 cases of STEMI (43\%) and 10 cases of NSTEMI $(10 \%)$. We observed a significant difference in the presence of complications in the two groups $(\mathrm{p}<0.001)$, Group $B$ patients with hyperglycemia had more complications. Significant difference was seen between the presence of heart failure, arrhythmias and shock in the two groups, with group B having more incidences of these $(\mathrm{p}<0.05)$. Significant difference in the outcome of the two groups was seen ( $p$ $=0.012$ ). We observed that out of 38 patients in Group B with hyperglycemia, there were 8 deaths (21.05\%) as compared to 3 deaths (4.84\%) in Group A patients.

\section{CONCLUSIONS}

We observed that the high prevalence (38\%) of stress in non-diabetic ACS patients is responsible for significantly more complications, more chances of heart failure, arrhythmia, shock and more deaths.

\section{KEY WORDS}

Acute Coronary Syndrome, Stress Hyperglycemia, Non-Diabetic Patients, Outcome
Corresponding Author: Dr. Radhika Ratanlal Bajaj, Department of Medicine, Krishna Hospital, Karad, Maharashtra, India. E-mail: bajajradhika44@gmail.com

DOI: $10.14260 / j e m d s / 2021 / 794$

How to Cite This Article:

Thorat ST, Bajaj RR, Rane VV, et al. Prognostic implication of stress induced hyperglycemia in non diabetic patients with acute coronary syndrome. J Evolution Med Dent Sci 2021;10(45):3931-3935, DOI: $10.14260 /$ jemds/2021/794

Submission 01-11-2021,

Peer Review 12-12-2021,

Acceptance 18-12-2021,

Published 28-12-2021.

Copyright (C) 2021 Sanjay Tukaram Thorat et al. This is an open access article distributed under Creative Commons Attribution License [Attribution 4.0 International (CC BY 4.0)] 


\section{BACKGROUND}

Hyperglycemia is a common finding during the admission of patients with acute coronary syndrome and is also a powerful predictor of survival, increasing the risk of immediate and long-term complications. ${ }^{1} \quad$ Epidemiological studies demonstrate that the relative risk of in-hospital death among patients with acute coronary syndrome who were unaware that they had DM and/or hyperglycemia at the time of the admission was 3.9 times higher than that of non-diabetics or healthy individuals. ${ }^{2,3}$

It is very essential to differentiate this stress hyperglycemia from the hyperglycemia in latent diabetics whose diabetes may have been unmasked by the stress of acute coronary syndrome. This is achieved by the estimation of glycosylated haemoglobin ${ }^{3}$ which reveals the blood sugar levels over the previous weeks and thus, identifies latent diabetics. ${ }^{4-7}$

Coronary artery disease (CAD) is the most common form of cardiovascular disease which is the leading cause of morbidity and mortality in both developed and developing world.8-10 The most important risk factors associated with CAD are diabetes, age, male gender, adverse lipid profile, smoking, hypertension, psychosocial factors and abdominal obesity. The clinical presentations of CAD include silent ischemia, stable angina pectoris, acute coronary syndrome, heart failure, and sudden cardiac death. $9,11,12$

Hyperglycemia is common and associated with markedly increased mortality rates in patients hospitalized with acute coronary syndromes. Despite the fact that several studies have documented this association, hyperglycemia remains underappreciated as a risk factor, and it is frequently untreated in ACS patients.

This is in large part due to limitations of prior studies, and the remaining critical gaps in our understanding of the relationship between hyperglycaemia and poor outcomes. First, there is currently no consensus about the precise glucose value (or range of values) that should be considered abnormal on admission. Second, there is no consensus about the most suitable method to initially measure and subsequently monitor blood glucose levels in the acute setting of ACS.

Third, the benefits of treating hyperglycaemia have not been established definitively, and the target value of blood glucose to be achieved with treatment remains undefined. The most important issue, however, is whether elevated glucose is a direct mediator of adverse outcomes in ACS patients or just a marker of greater disease severity. ${ }^{10}$

Epidemiological studies demonstrate that the relative risk of intra-hospital death among patients with acute coronary syndrome who were unaware that they had DM and/or hyperglycaemia at the time of the admission was 3.9 times higher than that of non-diabetics or healthy individuals. ${ }^{13}$

The data on the importance of controlling glucose in the hospital span diverse disciplines of medicine. Studies in the areas of stroke, MI, bypass surgery, wound and nosocomial infections all point to the tremendous potential to reduce the morbidity and mortality among hospitalized patients with hyperglycaemia. It is essential that hyperglycaemia is identified from the time of hospital admission and that therapy is implemented to achieve and maintain glucose levels as close to normal as possible regardless of patient's primary reason for admission or previous diabetes status. Identification of and therapeutic interventions to treat hyperglycaemia must be initiated in tandem with the presenting medical problem rather than days after admission. Existing data strongly suggest that an early and aggressive approach to the management of hyperglycaemia may reduce mortality, morbidity, excessive hospital stays and added costs. $^{14}$

\section{Objectives}

To evaluate prevalence of stress hyperglycaemia in nondiabetic acute coronary syndrome patients.

To evaluate the prognostic value of stress hyperglycaemia in determining the outcome in non-diabetic acute coronary syndrome patients.

\section{METHODS}

The present cross sectional study was conducted in the Department of Medicine, KIMS Hospital, Karad, over a period of one year. The Institutional Ethical Committee (IEC) approval was taken. The informed and written consent was taken from patients before enrolment for study.

\section{Inclusion Criteria}

Patients with acute coronary syndrome. Patients of ACS with complications

\section{Exclusion Criteria}

- Diabetic patients [Known case of DM or HbA1c > 6.5]

- Patients on drugs that cause hyper or hypoglycaemia

- Patients with prior history of infarction.

- Patients with ST elevation or depression due to causes other than ACS.

All patients of acute coronary syndrome who satisfy the inclusion criteria were selected for the study.

Detailed history of the patient was taken on admission. Acute coronary syndrome was diagnosed from:-

- History of chest pain

- Typical ECG findings

- $\quad$ Serum CPK-MB and troponin levels

Criteria for STEMI were any two of the following;

- Cardiac chest pain lasting at least 30 minutes,

- $>0.1 \mathrm{mV}$ ST elevation in at least one standard lead,

- $\quad>0.2 \mathrm{mV}$ ST elevation in two or more continuous chest leads, or

- New LBBB and biochemical markers of myocardial injury ${ }^{1}$

Unstable angina (UA) is defined as angina pectoris or equivalent ischemic discomfort with at least one of three features:

It occurs at rest (or with minimal exertion) usually lasting $\Rightarrow 10 \mathrm{~min}$. It is severe and of new onset (I.e. within the prior 4 to 6 weeks) and / or 
It occurs with a crescendo pattern (i.e. distinctly more severe, prolonged, or frequent than previously).

Parameters will include- Blood sugar levels, glycosylated $\mathrm{Hb}$ levels, lipid profile (VLDL, HDL, LDL, total cholesterol levels), myocardial markers (ECG, troponin levels).

Raw material was collected from trained medical staff using the medical records. Data was double checked for correctness, subsequently anonymised and transferred to the authors for data analysis.

\section{Sample Size Calculation}

According to a study conducted by Oswald GA et al. ${ }^{15}$ the proportion of stress hyperglycaemia was found to be $22.8 \%$, So, $\mathrm{p}=22.8 \%$

Using Cochran's formula for sample size (n) calculation,

$n=\frac{4 \times \mathrm{p} \times \mathrm{q}}{\mathrm{e} 2}$

Where, $\mathrm{p}=22.8 \%=0.228$

$\mathrm{q}=1-\mathrm{p}=0.772$

Taking e, absolute error of $10 \%, \mathrm{e}=0.1$

So,

$n=\frac{4 \times 0.228 \times 0.772}{0.1 \times 0.1}$

$\mathrm{n}=70.40 \approx 71$

A minimum of 71 patients were to be included in the study, we got 100 patients satisfying our study inclusion criteria so we included all of them in our study.

Sample size, $\mathrm{n}=100$.

\section{Statistical Analysis}

Data was collected using a semi structured pretested questionnaire, data was entered in Microsoft Excel 2013 and represented in frequencies and percentages, charts and graphs. Appropriate statistical tests are applied using SPSS software version 23 for analysis. Chi square test is used for association between the study variables. Statistical significance is considered at $\mathrm{p}<0.05$.

\section{RESULTS}

We analyzed a total of 100 patients of acute coronary syndrome satisfying our study inclusion criteria and we categorized the patients into two groups, Group A were normoglycaemic patients and Group B were acute phase hyperglycemic patients with blood sugars more than 160 . There were 62 cases (Group A - 62\%) with normal blood sugars and 38 cases (Group B - 38\%) with raised blood sugars.

\begin{tabular}{|cccc|}
\hline Age Groups (Yr) & Group A & Group B & Total \\
$<40$ years & 7 & 7 & 14 \\
41 to 60 & 20 & 12 & 32 \\
61 to 80 & 28 & 18 & 46 \\
81 to 100 & 7 & 1 & 8 \\
Total & $\mathbf{6 2}$ & $\mathbf{3 8}$ & $\mathbf{1 0 0}$ \\
\hline Table 1. Age Group Wise Distribution of the Study Participants \\
\hline $\mathrm{X}^{2}=3.09$, df $=2, \mathrm{p}=0.378$ & \multicolumn{4}{|l}{} \\
\hline
\end{tabular}

No significant difference in age distribution in the two groups was seen. $(\mathrm{p}>0.05)$
Most common age group was 61 to 80 years with 46 participants $(46 \%)$ followed by 41 to 60 years with 32 participants (32\%). Mean age was $65.23 \pm 21.05$ years.

\begin{tabular}{|cccc|}
\hline Risk Factors & Group A (62) & Group B (38) & P Value \\
\hline Smoking & 16 & 11 & 0.73 \\
Alcohol & 13 & 8 & 0.99 \\
Sedentary life style & 14 & 9 & 0.89 \\
Hypertension & 19 & 12 & 0.92 \\
\hline \multicolumn{2}{|c|}{ Table 2. High Risk Factors Present in the Two Groups } \\
\hline $\mathrm{p}>$ 0.05, Not significant & \\
\hline
\end{tabular}

No significant difference in risk factor distribution in the two groups was seen. $(\mathrm{p}>0.05)$.

16 out of 62 cases (25.81\%) in Group A were alcoholic while 11 out of 38 cases (28.95\%) in Group B were alcoholic. Alcoholism was seen in 13 cases (29.97\%) from Group A and in 8 cases $(21.05 \%)$ from Group B. Sedentary life style was observed in 14 cases (22.58\%) from Group A and in 9 cases from Group B (23.68\%). Hypertension was seen in 19 cases (30.65\%) from Group A and in 12 cases from Group B $(31.58 \%)$.

\begin{tabular}{|cccc|}
\hline Type of ACS & Group A (62) & Group B (38) & Total \\
NSTEMI & 6 & 4 & 10 \\
STEMI & 26 & 17 & 43 \\
UA & 30 & 17 & 47 \\
Total & $\mathbf{6 2}$ & $\mathbf{3 8}$ & $\mathbf{1 0 0}$ \\
\hline \multicolumn{4}{|c}{ Table 3. Type of ACS in the Two Groups } \\
\hline $\mathrm{X}^{2}=0.127, \mathrm{df}=2, \mathrm{p}=0.93$ & \multicolumn{3}{c}{} \\
\hline
\end{tabular}

No significant difference in type of ACS distribution in the two groups was seen.

We had 47 cases of unstable angina (UA - 47\%) followed by 43 cases of STEMI (43\%) and 10 cases of NSTEMI (10\%).

6 out of 62 cases $(9.68 \%)$ in Group A were NSTEMI while 4 out of 38 cases $(10.53 \%)$ in Group B were NSTEMI. STEMI was seen in 26 cases (41.94\%) and in 17 cases from Group B (44.74\%). Unstable angina was seen in 30 cases from Group A (48.39\%) and in 17 cases from Group B (44.74\%).

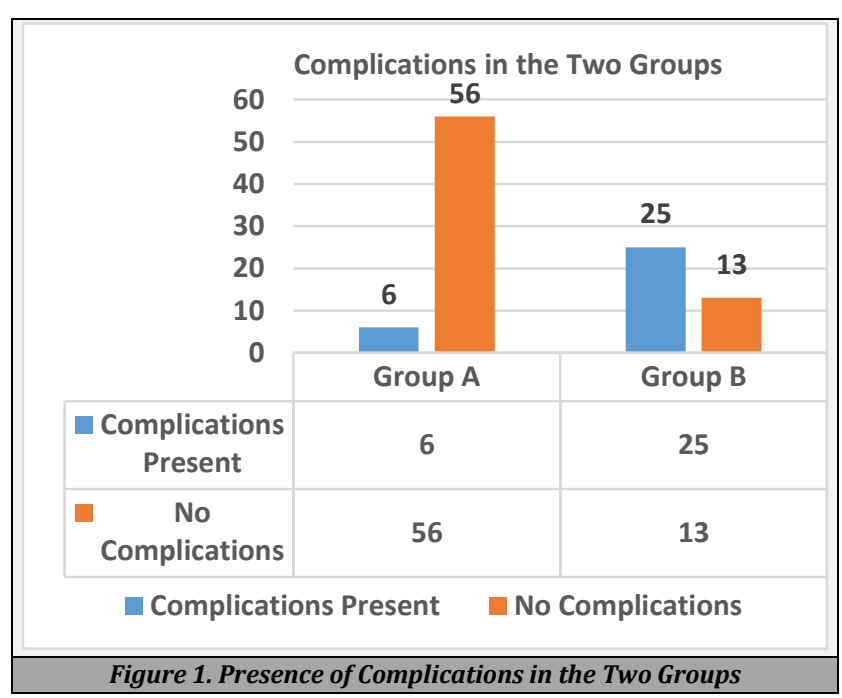

We observed a significant difference in the presence of complications in the two groups $(p<0.001)$, out of 38 Group B patients with hyperglycemia, 25 had complications $(65.79 \%)$ and out of 62 patients with normal sugars, only 6 had complications $(9.68 \%)$. 


\begin{tabular}{|cccc|}
\hline & Group A (62) & Group B (38) & P Value \\
Heart failure & 10 & 22 & $<0.001^{*}$ \\
Arrhythmia & 3 & 7 & $0.028^{*}$ \\
Shock & 5 & 11 & $0.006^{*}$ \\
\hline Table 4. Presence of Heart Failure, Arrhythmias and Shock in the Two \\
\multicolumn{4}{|c|}{ Groups } \\
\hline${ }^{*} \mathrm{P}<0.05$, Significant & \multicolumn{3}{l}{} \\
\hline
\end{tabular}

Significant difference was seen between the presence of heart failure, arrhythmias and shock in the two groups $(\mathrm{p}<0.05)$.

We observed that in the 38 patients with hyperglycemia, that is Group B, heart failure was seen in 22 cases (57.89\%), as compared to 10 cases in Group A (16.13\%). In Group B, arrhythmia was seen in 7 cases $(18.42 \%)$ as compared to 3 cases in Group A. In Group B shock was seen in 11 cases $(28.95 \%)$ as compared to 5 cases $(8.06 \%)$ in Group A. The overall presence of heart failure, arrhythmias and shock was seen significantly more in patients with hyperglycemia that is Group B patients.

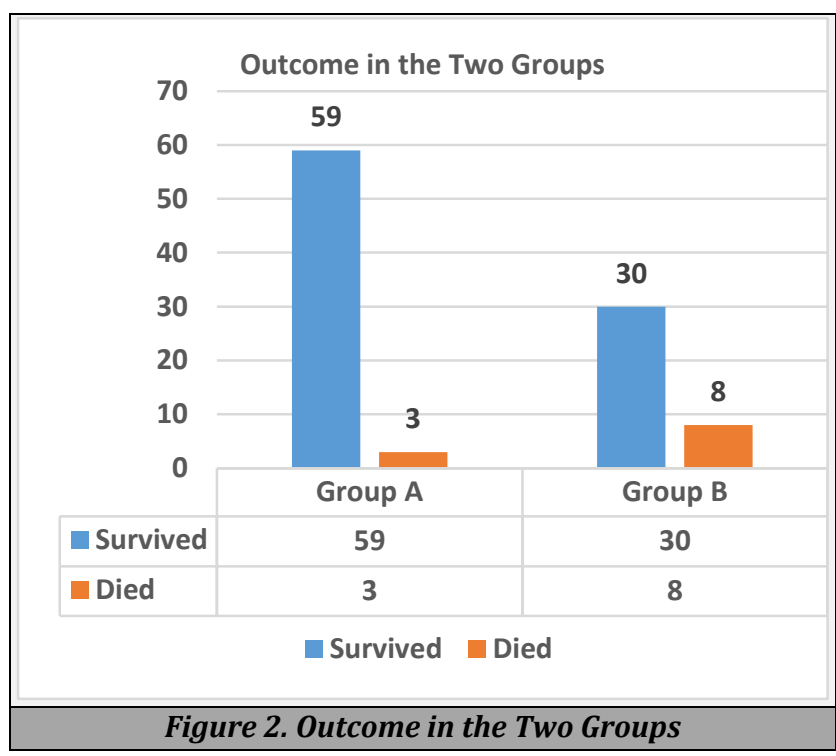

Significant difference in the outcome of the two groups was seen $(\mathrm{p}=0.012)$.

We observed that out of 38 patients in Group B with hyperglycemia, there were 8 deaths $(21.05 \%)$ as compared to 3 deaths (4.84\%) in Group A patients. We observed that the acute hyperglycemia in ACS patients is responsible for significantly more complications, more chances of heart failure, arrhythmia and shock and more deaths as compared to normoglycemic patients with ACS.

\section{DISCUSSION}

We had 38 patients (38\%) with stress induced hyperglycemia.

Similarly Zhou Na and Li Zhen ${ }^{13}$ in 2014 found 37\% prevalence of stress hyperglycemia in AMI patients who were not diabetics.

Also Nordin C et al.14 in a retrospective study found 35\% of patients of acute coronary syndrome having stress hyperglycemia without evidence of prior diabetes mellitus.

Oswald et al. ${ }^{15}$ measured HbA1C, cortisol concentration from 27 non-diabetic acute AMI patients. Through multiple regression analysis, they reported that cortisol, adrenaline, noradrenaline are major factor to determine glucose level on admission. However, it is still unclear whether this endocrine reaction is caused by excessive myocardial infarction or severe myocardial malfunction or both of them. Although the exact mechanism is still unknown, hyperglycemia in acute AMI induces thrombus formation through platelet activity, aspirin resistance, and impaired fibrinolysis. Hyperglycemia in acute AMI is known to be related with no reflow in AMI and pre inflammation change through increase of endothelial dysfunction, oxidative stress, cytokine adhesion molecule production no reflow. ${ }^{16-18}$

Most common age group was 61 to 80 years with 46 participants $(46 \%)$ followed by 41 to 60 years with 32 participants (32\%). Mean age was $65.23 \pm 21.05$ years.

No significant difference in age distribution or gender distribution in the two groups was seen. ( $p>0.05)$.

Abbas Ali Mansour et al. ${ }^{19}$ in 2011 found no significant difference in the mean age of the patients with stress hyperglycemia, diabetic and normoglycemic. This is in concordance with our study. Our findings are consistent with the findings of the other workers, like Taysir et al. in 2009 Marfella et al. in 2003 and Ameer et al. in 2010, who also found gender was not a significant factor for stress hyperglycemia. No significant difference in risk factor distribution in the two groups was seen. ( $p>0.05)$

Similarly, Abbas Ali Mansour et al. ${ }^{19}$ in 2011 in their study found no statistically significant difference in the history of smoking in different groups. This is in agreement with our study. Taysir Garadah et al. ${ }^{20}$ in 2009 in their study found no statistically significant difference in the history of smoking and hypertension in different groups. This is in concordance with our study. We had 47 cases of unstable angina (UA $47 \%$ ) followed by 43 cases of STEMI (43\%) and 10 cases of NSTEMI (10\%).

No significant difference in type of ACS distribution in the two groups was seen. ( $p>0.05)$ We observed a significant difference in the presence of complications in the two groups ( $\mathrm{p}<0.001$ ), out of 38 Group B patients with hyperglycemia, 25 had complications $(65.79 \%)$ and out of 62 patients with normal sugars, only 6 had complications (9.68\%).

Significant difference was seen between the presence of heart failure, arrhythmias and shock in the two groups $(\mathrm{p}<0.05)$.

We observed that in the 38 patients with hyperglycemia, that is Group B, heart failure was seen in 22 cases (57.89\%), as compared to 10 cases in Group A (16.13\%). In Group B, arrhythmia was seen in 7 cases (18.42\%) as compared to 3 cases in Group A and In Group B shock was seen in 11 cases $(28.95 \%)$ as compared to 5 cases $(8.06 \%)$ in Group A. The overall presence of heart failure, arrhythmias and shock was seen significantly more in patients with hyperglycemia that is Group B patients. Also Meisinger C et al. ${ }^{21}$ in 2006 showed admission hyperglycemia was also associated with complications during hospital stay. Significant difference in the outcome of the two groups was seen $(p=0.012)$.

We observed that out of 38 patients in Group B with hyperglycemia, there were 8 deaths $(21.05 \%)$ as compared to 3 deaths (4.84\%) in Group A patients.

EJ Kim et al. ${ }^{22}$ observed that the non-diabetic patients with hyperglycemia had significantly higher mortality $(17.4 \%)$ compared to diabetic patients $(7.2 \%, \mathrm{p}<0.001)$. 
Also Taysir Garadah et al..$^{20}$ in 2009 found that the stress hyperglycemia on admission is a powerful predictor of increased major adverse events, hospital mortality and cardiac failure in patients with acute coronary syndrome. This is in keeping with the findings of our study.

\section{CONCLUSIONS}

We observed that the high prevalence (38\%) of stress hyperglycemia i.e. admission blood sugar level $\geq 160 \mathrm{mg} / \mathrm{dl}$ in non-diabetic ACS patients is responsible for significantly more complications, more chances of heart failure, arrhythmia, shock and more deaths as compared to normoglycaemic patients with ACS. We observed no significant difference between age, gender, risk factors and type of ACS in two groups of patients.

Data sharing statement provided by the authors is available with the full text of this article at jemds.com.

Financial or other competing interests: None.

Disclosure forms provided by the authors are available with the full text of this article at jemds.com.

\section{REFERENCES}

[1] Ferreira JA, Baptista RM, Monteiro SR, et al. Admission hyperglycemia and all-cause mortality in diabetic and non-diabetic patients with acute myocardial infarction: a tertiary center analysis. Intern Emerg Med 2021;16(8):2109-19.

[2] Alavi-Moghaddam M, Parsa-Mahjoob M, Ghodssighassemabadi $\mathrm{R}$, et al. Association of admission blood glucose level with major adverse cardiac events in acute coronary syndrome; a cohort study. Arch Acad Emerg Med 2019;7(1):e26.

[3] Petursson P, Herlitz J, Caidahl K, et al. Admission glycaemia and outcome after acute coronary syndrome. Int J Cardiol 2007;116(3):315-20.

[4] Kodilkar J, Kolge M, Chafekar N, et al. Study to evaluate correlation of blood sugar level and glycosylated haemoglobin at the time of admission with severity of acute coronary syndrome in diabetic patients. MVP J Med Sci 2018;5(1):39-48.

[5] Dar MI, Beig JR, Jan I, et al. Prevalence of type 2 diabetes mellitus and association of $\mathrm{HbA1c}$ with severity of coronary artery disease in patients presenting as nondiabetic acute coronary syndrome. Egypt Heart J 2020;72(1):66.

[6] Rajuri V, Priyanka N. A study on glycaemic status impact in acute coronary syndrome. Journal of Evolution of Medical and Dental Sciences 2019;8(25):1983-8.

[7] Chaudhary V, Aundhakar S, Afzalpurkar S, et al. Evaluation of admission time hyperglycaemia in acute coronary syndrome patients. Journal of Evolution of Medical and Dental Sciences 2017;6(20):1639-42.
[8] Kumar AS, Sinha N. Cardiovascular disease in India: a 360 degree overview. Med J Armed Forces India 2020;76(1):1-3.

[9] Malakar AK, Choudhury D, Halder B, et al. A review on coronary artery disease, its risk factors, and therapeutics. J Cell Physiol 2019;234(10):16812-23.

[10] Olinic DM, Spinu M, Olinic M, et al. Epidemiology of peripheral artery disease in Europe: VAS educational paper. Int Angiol 2018;37(4):327-34.

[11] Hajar R. Risk factors for coronary artery disease: historical perspectives. Heart Views 2017;18(3):109-14.

[12] van Rosendael AR, Bax AM, Smit JM, et al. Clinical risk factors and atherosclerotic plaque extent to define risk for major events in patients without obstructive coronary artery disease: the long-term coronary computed tomography angiography CONFIRM registry. Eur Heart J Cardiovasc Imaging 2020;21(5):479-88.

[13] Z, Li Zhen L. GW25-e0795 Relationship between stress hyperglycemia and in hospital mortality and complications in patients with acute myocardial infarction. JA Crit Cardiol 2014;64(16_Supplement) C126.

[14] Nordin C, Amiruddin R, Rucker L, et al. Diabetes and stress hyperglycemia associated with myocardial infarctions at an urban municipal hospital: prevalence and effect on mortality. Cardiol Rev 2005;13(5):223-30.

[15] Oswald GA, Smith CC, Betteridge DJ, et al. Determinants and importance of stress hyperglycaemia in non-diabetic patients with myocardial infarction. Br Med J (Clin Res Ed) 1986;293(6552):917-22.

[16] Le Guyader A, Pacheco G, Seaver N, et al. Inhibition of platelet GPIIb-IIIa and P-selectin expression by aspirin is impaired by stress hyperglycemia. J Diabetes Complications 2009;23(1):65-70.

[17] Williams SB, Goldfine AB, Timimi FK, et al. Acute hyperglycemia attenuates endothelium-dependent vasodilation in humans in vivo. Circulation 1998;97(17):1695-701.

[18] Esposito K, Nappo F, Marfella R, et al. Inflammatory cytokine concentrations are acutely increased by hyperglycemia in humans: role of oxidative stress. Circulation 2002;106(16):2067-72.

[19] Mansour AA, Wanoose HL. Acute phase hyperglycemia among patients hospitalized with acute coronary syndrome: prevalence and prognostic significance. Oman Med J 2011;26(2):85-90.

[20] Garadah TS, Kassab S, Al-Shboul QM, et al. The threshold of admission glycemia as a predictor of adverse events in diabetic and non-diabetic patients with acute coronary syndrome clinical medicine. Clin Med Cardiol 2009;3:2936.

[21] Meisinger C, Hörmann A, Heier M, et al. Admission blood glucose and adverse outcomes in non-diabetic patients with myocardial infarction in the reperfusion era. Int J Cardiol 2006;113(2):229-35.

[22] Kim EJ, Jeong MH, Kim JH, et al. Clinical impact of admission hyperglycemia on in-hospital mortality in acute myocardial infarction patients. Int J Cardiol 2017;236:9-15. 